\title{
A mho type phase comparator relay guideline using phase comparison technique for a power system
}

\author{
José Antonio González-Cueto Cruz ${ }^{1}$, Zaid García Sánchez ${ }^{2}$, Gustavo Crespo Sánchez ${ }^{3}$, \\ Hernán Hernández Herrera ${ }^{4}$, Jorge Iván Silva-Ortega ${ }^{5}$, Vicente Leonel Martínez Díaz ${ }^{6}$ \\ ${ }^{1,2,3}$ Center of Energy and Environmental Studies Department, Universidad de Cienfuegos, Cuba \\ ${ }^{4,6}$ Universidad Simón Bolívar, Facultad de Ingenierías, Barranquilla, Colombia \\ ${ }^{5}$ Departamento de Energía, Universidad de la Costa, Barranquilla, Colombia
}

\section{Article Info}

Article history:

Received Nov 6, 2019

Revised Aug 17, 2020

Accepted Sep 24, 2020

\section{Keywords:}

Distance relays

Electric power network analysis

Mid-term stability

Mho phase comparator

\begin{abstract}
This paper presents a mho distance relay simulation based on the phase comparison technique using a typical electrical power systems analysis software for two cases: when the operation state is close to the static voltage limit and during a dynamic perturbation in the system. The paper evaluates the impedance variations caused by complex voltage values, the mho polarization, and the comparator operating region into the complex plane. In addition, the paper found the information for the dynamic perturbations from the outputs considering a mid-term stability program. The simulation of the mho-phase comparator in the static voltage proximity limit detects unit distance elements with impedance measured close to reach the threshold in the steady-state. Dynamic mho simulations in the complex plane are successfully tested by plotting time phase difference curves on the comparator input signals. Relay programmers can use these curves to analyze other phase comparators applications and the corresponding models in the complex plane.
\end{abstract}

This is an open access article under the CC BY-SA license.

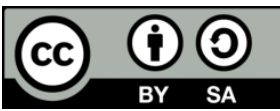

Corresponding Author:

Hernán Hernández Herrera

Facultad de Ingeniería

Universidad Simón Bolívar

Barranquilla, Colombia

Email: hernan.hernandez@unisimonbolivar.edu.co, hernanhh813@gmail.com

\section{NOMENCLATURE}

$\underline{S}_{1}, \underline{S}_{2}$ Phase comparator complex input voltage signals.

$\underline{k}_{1}, \underline{k}_{2}$,Phase comparator complex constants which

$k_{3}, k_{4}$ define the threshold to operating conditions.

$\underline{k}_{p} \quad$ Complex constant to adjust.

$\underline{S}_{2} \quad$ Value in a mho phase comparator.

$\underline{V} \quad$ Phase comparator input complex voltage. $\theta_{1}, \theta_{2}$ Angular Threshold values in a phase comparator setup.

$\underline{Z} \quad$ Phase comparator input complex impedance measured.

$\underline{\boldsymbol{A}}, \underline{B}$ Phase comparator complex constant impedances.

$\underline{Z}_{r} \quad$ Complex replica impedance of mho phase comparator, equal to the reached by the relay.

$\underline{V}_{p} \quad$ Complex signal voltage of mho phase comparator used for increase reliability polarization voltage. 
$\underline{I} \quad$ Phase comparator input complex current.

$\theta \quad$ Angular difference between $\underline{S}_{1}, \underline{S}_{2}$.

$\lambda$ Angular difference between the complex constants.

$P \quad$ Active power load.

$p v$ Active power load voltage exponent.

$J_{R} \quad$ Reduced Jacobian matrix of node reactive power injections with respect node voltage modules.

$J_{Q \theta} \quad$ Jacobian sub-matrix of node reactive power injections partial derivatives with respect node voltage angles.

$J_{P V} \quad$ Jacobian sub-matrix of node active power injections, partial derivatives with respect node voltage modules.

$\Delta V$ Incremental variations vector of complex positive sequence node voltages.

$Y_{S R} \quad$ Positive sequence mutual admission module from the sending end to the receiving end on one line.

$V_{R} \quad$ Positive sequence voltage module on a receiving end line.

$\underline{I}_{S} \quad$ Complex positive sequence current in line sending end.

$\underline{Y}_{S R} \quad$ Complex mutual admittance of positive sequence from sending end to receiving end in a transmission line.

$\underline{Y}_{S S} \quad$ Complex positive sequence self-admittance in a line sending end line.

$\underline{V}_{S} \quad$ Complex positive sequence voltage in line sending end.

$\underline{Z}_{S M} \quad$ Complex impedance measured in line sending end at balanced conditions.

$\underline{Y}_{S H} \quad$ Complex positive sequence shunt admittance of a line.

C Mho circle center point in the complex plane.

$\underline{Z}_{i j}(k) \quad$ Complex line to line impedance measured in network line end at instant $k$.

$\underline{V}_{i j}(k)$ Positive sequence complex line to line voltage $i j$ for a network line end at instant $k$.

$\underline{V}_{i}(k)$ Complex phase voltage $i$ of positive sequence in a network line end at instant $k$.

$\left[V_{i}\right]^{012}$ Complex phase voltage vector of node $\mathrm{i}$ in sequence components.

$\left[V_{B U S}\right]^{012}$ Complex bus voltage vector for a network in sequence components.

$\left[\underline{I}_{B U S}\right]^{012}$ Complex bus current vector for a network in sequence components.

$\left[Y_{S R}\right]^{012}$ Complex mutual admittance sub-matrix of a line from sending end to receiving end in sequence components.

$\left[Y_{R R}\right]^{012}$ Complex self admittance, sub-matrix of a

$\left.\underline{Y}_{R R}\right] \quad$ line receiving end in sequence components.

$\left[I_{R}\right]^{a b c}$ Complex phase current vector of a line receiving end in-phase components.

$\left[V_{R}\right]^{012}$ Complex phase voltage vector of a line receiving end in sequence components.
$\underline{Z}_{p} \quad$ Complex impedance related to $\underline{V}_{p}$.

$\underline{A}_{p} \quad$ Complex variable impedance of mho phase comparator related to $\underline{Z}_{p}$.

$V \quad$ Node voltage module of positive sequence.

$Q \quad$ Reactive power load.

$q v \quad$ Reactive power load voltage exponent.

$J_{Q V}$ Jacobian sub-matrix of node reactive power injections partial derivatives with respect node voltage modules.

$\boldsymbol{J}_{\boldsymbol{P} \boldsymbol{\theta}}^{-1}$ Inverse Jacobian sub-matrix of node active power injections, partial derivatives with respect node voltage angles.

$\Delta Q \quad$ Incremental variations vector of node reactive power injections.

$P_{\text {Max }} \quad$ Maximum active power received of a line at steady state operation for known end voltages.

$V_{S} \quad$ Positive sequence voltage module of a line sending end.

$G_{R R} \quad$ Positive sequence self conductance of a line receiving end.

$\underline{I}_{R} \quad$ Complex positive sequence current in line receiving end.

$\underline{Y}_{R S} \quad$ Complex mutual admittance of positive sequence from receiving end to sending end of a line.

$\underline{Y}_{R R} \quad$ Complex positive sequence self-admittance of a line receiving end.

$\underline{V}_{R} \quad$ Complex positive sequence voltage in line receiving end.

$\underline{Z}_{R M} \quad$ Complex impedance measured in line receiving end at balanced conditions.

$\underline{Y}_{S E R} \quad$ Complex positive sequence series admittance of a line.

$R \quad$ Radius of a mho circle.

$\underline{Z}_{p h}(k) \quad$ Complex phase impedance measured in line end at instant $k$.

$\underline{I}_{i}(k)$ Complex line current $i$ of positive sequence in a network line end at instant $k$.

$\left[\underline{V}_{i}\right]^{a b c}$ Complex phase voltage vector of node $\mathrm{i}$ inphase components.

$\left[T_{S}\right]$ Transformation matrix of sequence components to phase components.

$\left[\underline{Z}_{B U S}\right]^{012}$ Complex bus impedance matrix for a network in sequence components.

$\left[\underline{Y}_{S S}\right]^{012}$ Complex self admittance sub-matrix of a

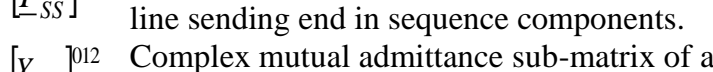

$\left[\underline{Y}_{R S}\right]^{12}$ line from receiving end to sending end in sequence components.

$\left[I_{S}\right]^{a b c}$ Complex phase current vector of a line sending end in-phase components.

$\left[V_{S}\right]^{012}$ Complex phase voltage vector of a line sending an end in sequence components. 


\section{INTRODUCTION}

Utilities and manufacturers recognize protection scheme modeling for power systems as a useful tool to analyze their operation and parameters [1,2]. Researchers developed the phase-comparison method many years ago for transmission-lines scheme protections [3, 4]. However, optimal allocation and dynamic design require feature to improve $[2,5]$. Relay simulation in Power Systems is an activity recognized by technical literature thirty years ago as an option to analyze its operation and adjusting parameters [6], transient analysis [7], and modeling using computer sciences for distance relays [8]. Since then, relays modeling is a complementary tool to prove and adjust operation schemes $[9,10]$. The mho type distance relay development and analysis consider several polarization methods for transmission-lines scheme protections [10-13]. There are precedents in digital software development to analyze the protection response in the complex plane during a short-circuit event [14-16]. And other topics related with supervision and modeling such as those referred to in $[17,18]$.

Based on the fundamentals of the phase comparators, this paper presents a new technique to determinate the displacement operating zone in a mho relay complex plane is a novel aspect, considering that the operating threshold of the mho in a complex plane is the polar characteristic on a circle. The research introduces relevant principles and techniques used in the software design. The paper also describes the main equations used by the software and the output results used to get the protection zones characteristics and the impedance measured by the phase comparator. The paper includes two study cases to assess the simulation of the protection plotting the time phase difference curves for a comparator input signals. These curves must have phase difference values for operating conditions, these values have a range between -90 and 90 degrees, in the moment when the corresponding measured impedances are above or within the operation circle in the complex plane. The presence of symmetric and asymmetric disturbances such as short circuits, open conductors in a transmission-lines and power oscillations considers the effects of polarization type and memory usage. Transmission lines are the case study used, but its applications as phase comparison also can be able to include cases considering protections with FACTS devices $[19,20]$, shunt compensation line protections [21], series compensation [22] and protections in networks with wind generators [23].

\section{RESEARCH METHOD}

\subsection{Two-input phase comparator}

The following equations are the input signals in a phase comparator in the most general case [3, 5, 15]:

$$
\begin{aligned}
& \underline{S}_{1}=\underline{k}_{1} \underline{V}+\underline{k}_{2} \underline{I} \\
& \underline{S}_{2}=\underline{k}_{3} \underline{V}+\underline{k}_{4} \underline{I}
\end{aligned}
$$

Phase comparator reaches the operating condition when both phase difference signals are in the following interval:

$$
\theta_{1} \leq \theta=\arg \left(\underline{S}_{1} / \underline{S}_{2}\right) \leq \theta_{2}
$$

where,

$\theta$ is the phase difference of $\underline{S}_{1}$ and $\underline{S}_{2}$.

$\theta_{1}$ and $\theta_{2}$ are constant magnitudes.

Replacing (1) and (2) into (3) gives an equivalent expression showed as follows:

$$
\theta_{1} \leq \theta=\arg \left(\frac{\underline{S}_{1}}{\underline{S}_{2}}\right)=\arg \left[\frac{\underline{k}_{1} \underline{V}+\underline{k}_{2} \underline{I}}{\underline{k}_{3} \underline{V}+\underline{k}_{4} \underline{I}}\right] \leq \theta_{2}
$$

If (4) is divided by $\underline{I}$, when its value is differing from zero, the equation is:

$$
\left.\theta_{1} \leq \arg \left[\frac{\underline{k}_{1} \underline{Z}+\underline{k}_{2}}{\underline{k}_{3} \underline{V}+\underline{k}_{4}}\right]=\arg [\underline{\underline{Z}-\underline{B}}] \underline{\underline{Z}}-\underline{A}\right]+\theta_{2}
$$

where, 


$$
\underline{A}=-\frac{\underline{k}_{4}}{\underline{k}_{3}}, \underline{B}=-\frac{\underline{k}_{2}}{\underline{k}_{1}}, \lambda=\arg \left[\frac{\underline{k}_{1}}{\underline{k}_{3}}\right]
$$

We can write (5) as:

$$
\theta_{1}-\lambda \leq \arg \left(\frac{\underline{Z}-\underline{B}}{\underline{Z}-\underline{A}}\right)=\arg \left(\frac{\underline{Z}_{S 1}}{\underline{Z}_{S 2}}\right) \leq \theta_{2}-\lambda
$$

where, $\quad \underline{Z}_{S 1}=\underline{Z}-\underline{B}$ and $\underline{Z}_{S 2}=\underline{Z}-\underline{A}$

The form and dimensions of a phase comparator characteristics in the complex Z-plane are given by the threshold operating conditions, using conditions in (7) to determine the equality. $\underline{k}, \theta_{1}$ and $\theta_{2}$ values defines the equality conditions. Constants $\mathrm{A}$ and $\mathrm{B}$ are the impedance values which determinate the characteristic in the complex plane, $\underline{A}=\underline{Z}-\underline{Z}_{S 2}$ and $\underline{B}=\underline{Z}-\underline{Z}_{S 1}$. Thus, the alternating currents and voltages amplitude and phase variations do not affect the comparator operating zone in the complex plane, while using computational tools the comparator can measure current and voltage.

\subsection{Mho phase comparator}

The following input signals to the phase comparator allow to obtain a mho type relay.

$$
\begin{aligned}
& \underline{S}_{1}=-\underline{V}+\underline{Z}_{r} \underline{I} \\
& \underline{S}_{2}=\underline{V}
\end{aligned}
$$

where, $\quad \underline{k}_{1}=-1, \underline{k}_{2}=\underline{Z}_{r}, \underline{k}_{3}=1$ and $\underline{k}_{4}=0$

The replica impedance $\underline{Z}_{r}$ is identical to the desired relay range. In this general case the mho type relay is self- polarized and its characteristic in the complex plane is a circle which pass through the origin when the limits and constants are:

$$
\theta_{1}-\lambda=90^{\circ}, \theta_{2}-\lambda=-90^{\circ}, \underline{A}=0, \underline{B}=\underline{Z}_{r}
$$

and,

$$
\theta_{1}=-90^{\circ}, \theta_{2}=90^{\circ}, \lambda=-180^{\circ}
$$

Hence, $\underline{Z}_{S l}$ and $\underline{S}_{l}$ have a phase difference of $180^{\circ}$.

This protection requires polarization signal $\underline{S}_{2}$ in order to provide a reliable reference angle. The selfpolarization does not suit when a bolted fault is too close to the signal, therefore adaptive polarization is a better option. It can be cross, combined or memory polarized [15, 24]. The first two fail on bold three-phase short circuits, only memory polarization solves the problem. With a mho relay with combined or dual polarization, the S2 signal describes the following math relation.

$$
\underline{S}_{2}=\underline{V}+\underline{k}_{p} \underline{V}_{p}
$$

While for the cross or simple polarization, the S2 signal is

$$
\underline{S}_{2}=\underline{k}_{p} \underline{V}_{p}
$$

The mho threshold operating conditions in the complex plane are:

$$
\theta_{1}-\lambda \leq \arg \left(\frac{\underline{Z}-\underline{Z}_{r}}{\underline{Z}_{S 2}}\right)=\arg \left(\frac{\underline{Z}-\underline{B}}{\underline{Z}-\underline{A}_{p}}\right) \leq \theta_{2}-\lambda
$$

In the mho $\underline{A}_{p}$ and $\underline{B}$ are also impedance values which determine the circle diameter in the complex plane. 


$$
\underline{B}=\underline{Z}_{r}
$$

In combined polarization

$$
\underline{Z}_{S 2}=\left(\underline{V}+\underline{k}_{p} \underline{V}_{p}\right) / \underline{I}=\underline{Z}+\underline{Z}_{p}
$$

and the variable impedance $\underline{A}_{p}$ in the complex plane is

$$
\underline{A}_{p}=\underline{Z}_{-} \underline{Z}_{S 2}=-\underline{Z}_{p}
$$

In simple polarization

$$
\begin{aligned}
& \underline{Z}_{S 2}=\underline{k}_{p} \underline{V}_{p} / \underline{I}=\underline{Z}_{p} \\
& \underline{A}_{p}=\underline{Z}-\underline{Z}_{S 2}=\underline{Z}-\underline{Z}_{p}
\end{aligned}
$$

The common dual and simple polarization signals are shown in [15]. Using positive sequence, polarization with memory is explained in [24]. Thus, the characteristic of self-polarized mho relay will always be static in the complex plane and equal for all phase and line relays with the same range. While for the combined polarization, the characteristics displaces from the origin according with the value of $\mathrm{Zp}$. For unbalanced conditions phase to ground and line to line characteristic protections are different. The simple polarization behavior in balanced operation and without memory action is identical to self-polarized. In unbalanced conditions or voltage variations under memory effect, the simple polarization characteristics displace from the origin. In unbalanced operation all phases and line characteristic protections are also different in the simple polarization. The following example describe these issues.

Figure 1(a) shows the complex plane considering three mho typical characteristics, M1 selfpolarized, M2 and M3 with a combined polarization and a $0.15|\mathrm{kp}|$ value. The last one has a reverse reach for M1, M2. M3 have an $80 \%$ modular reach in the Zl. For a three-phase fault in F, middle of the line, M1 and $\mathrm{M} 2$ currents are $\mathrm{I}=2 \mathrm{~V} / \mathrm{Zl}$ and $\mathrm{M} 3$ current is $\mathrm{I}=-2 \mathrm{~V} / \mathrm{Zl}$. The points Ap2 and Ap3 are matching because the M3 characteristic in Figure 1(a) draw a 180-phase shift in the complex plane for its reverse reach. When M1 and M2 operates, M3 is inactive. Table 1 shows the most important unit impedances while Figure 1(b) shows the input signal values and its phase shift and Table 1 describes each unit. The current angle measured by the comparators cause the phase shift between $\mathrm{Zp}$ and $\mathrm{Zr}$ and subsequent variation in the M2 and M3 diameters, resulting a shift of the characteristics.

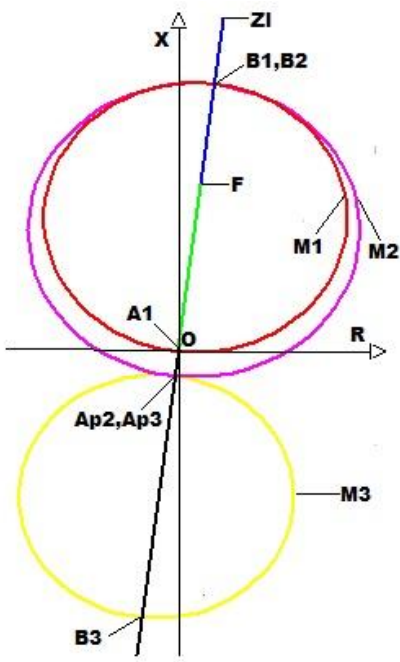

(a)

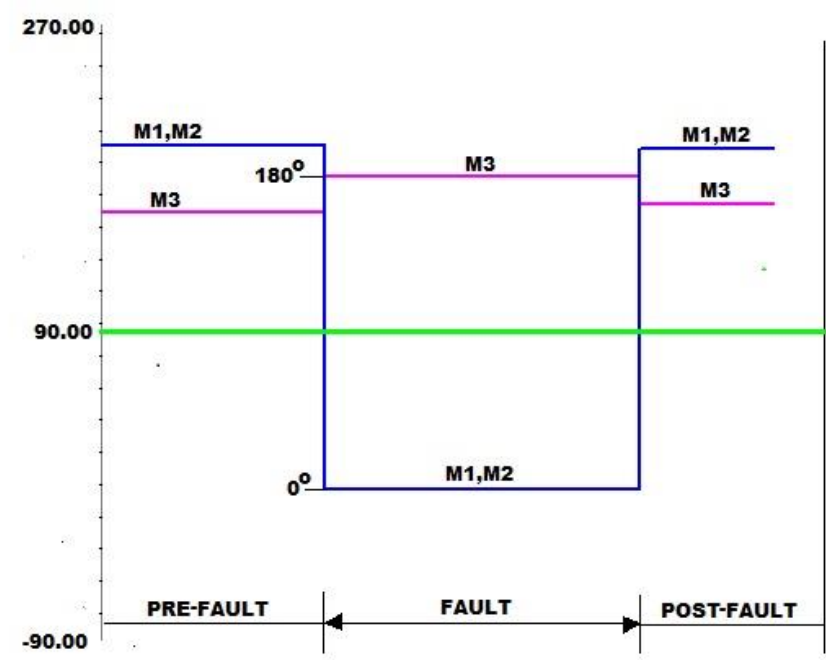

(b)

Figure 1. Input signals values and its phase shift of M1, M2 and M3 units, (a) complex plane, (b) arguments $\left(\underline{S}_{1} / \underline{S}_{2}\right)$ 
Table 1. Values of the input signals and important impedances of M1, M2 and M3 units

\begin{tabular}{cccccccc}
\hline Unit & $\underline{S}_{l}$ & $\underline{S}_{2}$ & $\operatorname{Arg}\left(\underline{S}_{l} / \underline{S}_{2}\right)$ & $\underline{Z}_{r}$ & $\underline{Z}_{p}$ & $\underline{A}_{P}$ & $\underline{B}$ \\
\hline M1 & $0.6 \underline{V}$ & $\underline{V}$ & $0^{0}$ & $0.8 \underline{Z}_{l}$ & 0 & 0 & $0.8 \underline{Z}_{l}$ \\
M2 & $0.6 \underline{V}$ & $\underline{V}$ & $0^{0}$ & $0.8 \underline{Z}_{l}$ & $0.15 \underline{Z}_{l}$ & $-0.15 \underline{Z}_{l}$ & $0.8 \underline{Z}_{l}$ \\
M3 & $-1.6 \underline{V}$ & $\underline{V}$ & $180^{0}$ & $0.8 \underline{Z}_{l}$ & $-0.15 \underline{Z}_{l}$ & $-0.15 \underline{Z}_{l}$ & $-0.8 \underline{Z}_{l}$ \\
\hline
\end{tabular}

\section{SYSTEM ANALYSIS USINGCOMPUTATIONAL TOOLS}

\subsection{Static voltage stability}

Utilities and Relay programmers can find the network operation limit in a steady state, increasing the load in different selective forms. The software implements three algorithms: i) Fast uncoupled newtonraphson algorithm (NR) [25]; ii) Formal NR algorithm [26, 27] and; iii) Continuous power flow algorithm based on NR [28]. Also, the software plots the active and reactive power vs voltage and load angle from the algorithms output. Results shows the power load flows for the critical point.

The voltage collapse point is the maximum load point transferred into the system for constant power loads vs. voltage. The algorithms continue increasing the burden while the final power for each iteration exceeds the previous iteration. The critical point is in vicinity with non convergence power flow point. The voltage vs the constant active power characteristic is a perpendicular straight line to the power axis. The maximum load and the static bifurcation point require another proof when considering loads varying with the voltage. Equations showed in (18) represents loads below with exponents $p v$ and $q v$ values different from zero $[29,30]$. The sub-index 0 identifies the initial state of the algorithm.

$$
P=P_{0}\left(\frac{V}{V_{0}}\right)^{p v}, Q=Q_{0}\left(\frac{V}{V_{0}}\right)^{q v}
$$

Searching for the critical point solutions, it can be found a point with greater load power than the previous one under unstable with voltage dependent loads. Figure 2 shows this situation near the critical point. Point $\mathrm{A}$ is not the maximum point and for an increment in the load the software can converge like in a B point, which could be an unstable point. We can find other solutions when the maximum load and the bifurcation point are different. A sensitivity test is a good solution in these cases. [27, 29, 31].

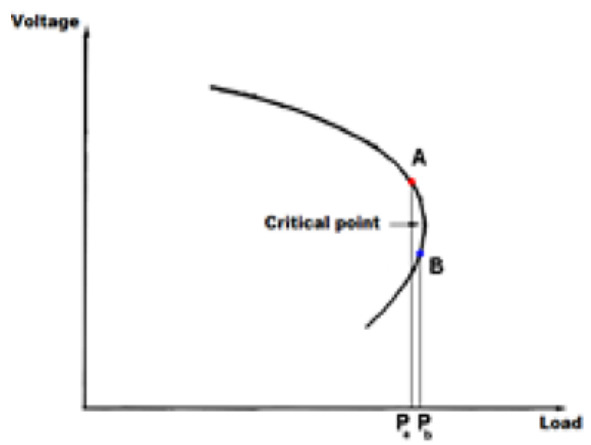

Figure 2. Critical point vicinity

The solution point is unstable in the sensitivity test of one term on the main diagonal if $J_{R}^{-1}$ is negative. These terms are the total derivatives of the voltages regarding to the reactive power variations. The matrix $J_{R}$ is a reduced matrix found by the following matrix equations, where $J_{P \theta}, J_{P V}, J_{Q \theta}$ and $J_{Q V}$ are network jacobian sub-matrices.

$$
\begin{aligned}
& J_{R}=J_{Q V}-J_{Q \theta} J_{P \theta}^{-1} J_{P V} \\
& \Delta Q=J_{R} \Delta V \\
& \Delta V=J_{R}^{-1} \Delta Q
\end{aligned}
$$


The software can make a modal analysis and find the eigenvalues using a reduced matrix $J_{R}$ with a critical state variable value. The participation factors of nodes, branches and generators can be found for the smaller eigenvalues and the network critical elements. This analysis is a measure of the vicinity to the critical point and the branches with high participations are weak links or are heavily loaded [27]. The line with the highest active power percent transferred is determined by (22). These elements are useful for contingency selection and it allow to identify the protections closer to their reach threshold values in the critical state.

$$
P_{\text {Max }}=Y_{S R} V_{S} V_{R}-G_{R R} V_{R}^{2}
$$

The following equation allow to find the branches currents:

$$
\left[\begin{array}{l}
\underline{I}_{S} \\
\underline{I}_{R}
\end{array}\right]=\left[\begin{array}{cc}
\underline{Y}_{S S} & -\underline{Y}_{S R} \\
-\underline{Y}_{R S} & \underline{Y}_{R R}
\end{array}\right]\left[\begin{array}{l}
\underline{V}_{S} \\
\underline{V}_{R}
\end{array}\right]
$$

The results in this computational tool considers balanced phases, and voltages on each end of the line allowing to find the impedance values.

$$
\begin{aligned}
& \underline{Z}_{S M}=\underline{V}_{S} /\left[\underline{Y}_{S H} \underline{V}_{S}+\underline{Y}_{S E R}\left(\underline{V}_{S}-\underline{V}_{R}\right)\right] \\
& \underline{Z}_{R M}=\underline{V}_{R} /\left[\underline{Y}_{S H} \underline{V}_{R}+\underline{Y}_{S E R}\left(\underline{V}_{R}-\underline{V}_{S}\right)\right]
\end{aligned}
$$

Hence, the impedance values above for each stable stage solution procedure from the initial condition to the critical point plot a curve in the complex plane. As shown in (13) describe the mho phase comparators characteristics in the complex plane at point $\underline{B}$ and at point $\underline{A}_{p}$ by (14) to (17). Depending on the polarization type, $\underline{B}$ and $\underline{A}_{p}$ can also be found by the voltages of both ends of each line for the balanced conditions in the critical state. These characteristics are circles with center in (26) and radio in (27).

$$
\begin{aligned}
& \underline{C}=\left(\underline{B}+\underline{A}_{p}\right) / 2 \\
& R=\left|\left(\underline{B}+\underline{A}_{p}\right)\right| / 2
\end{aligned}
$$

The software shows the impedance measured curves and all the zone characteristics of mho phase comparators in the complex plane. This can also be done for all selected lines ends.

\subsection{Mid-term synchronous stability}

The numerical method used in the mid-term stability program for the differential equation solutions is the implicit trapezoidal integration method with a $\mathrm{Z}_{\mathrm{Bus}}$ load flow for the network solution [26, 32]. The main system elements and its interrelationships are shown in Figure 3.

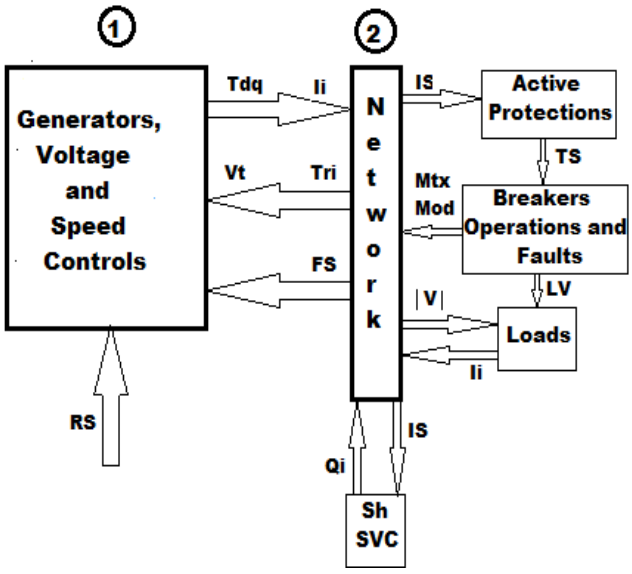

Figure 3. Element interrelationships in mid-term stability program 
Figure 3 shows the interchange between the networks with:

- Generators and its controls. The network and generators interactions are dq-ri variable transformations, Tdq-Tri; and reference and feedback signals, RS, FS, for the controls. The synchronous generators represent the simplest flux model, constant emf, to $\mathrm{d}$ and $\mathrm{q}$ sub-transient effects with saturation. The prime motors may be steam, gas and hydraulics turbines and internal combustion motors. Also, it can simulate Eolic turbines with induction generators and photovoltaic generation.

- Active protections, with input signals and tripping signals, IS and TS. These protections during simulation can measure frequency, frequency variation, voltage, active and reactive power, impedances and inversion of active power.

- Breaker operations and simulated faults, with matrix modification and-or load variation, MtxMod and LV.

- Loads, with its current injections Ii under voltage variations $|\mathrm{V}|$, frequency and load shedding or load reclosing by the input signals, $\mathrm{LV}$.

- Shunt SVCs, with their input control signals IS and output reactive power injections Qi.

The $3 \times 3$ sequence component elementary sub-matrix for each self or mutual admittances of the network form the $\mathrm{Y}_{\text {Bus }}$ matrix terms. Thus, a shunt fault is represented by a fictitious admittance load insertion in the matrix corresponding node, like in Figure 4. The fault is determined by the four admittances values and the $3 \times 3$ phase component submatrix is obtained by Kron elimination on the neutral node. Then the resulting $3 \times 3$ fault sequence components sub-matrix is found by the transformation:

$$
\left[Y_{f}\right]^{012}=\left[T_{S}\right]^{-1}\left[Y_{f}\right]^{a b c}\left[T_{S}\right]
$$

where,

$\left[T_{S}\right] \quad:$ is the transformation matrix for sequence components to phase components.

$\left[Y_{f}\right]^{a b c} \quad:$ is the fault submatrix in-phase components.

$\left[Y_{f}\right]^{012}$ : is the fault submatrix in sequence components.

Three series admittances represent series faults. This method is easier than others to simulate:

- Simultaneous symmetrical or asymmetrical faults in various network points.

- Shunt faults in internal points of the line.

- Breaker switching.

Active protections or a programmed switching may trip breakers by a selection form as is shown in Figure 5. The mutual inductance effect of zero sequence in transmission lines can be simulated in asymmetrical faults cases related with ground. The results in the software are given in graphical and tabular form with optional configurations. One graphic shows the impedance variation curves measured modules and the angle measured from the apparent power phasors versus time, in both line ends. The aim is to detect those protections, which can measure impedances or oscillatory values near their reach. These values judge against the value of the line impedance in each simulation instant. The phase difference between the powers vectors are verified all instants.

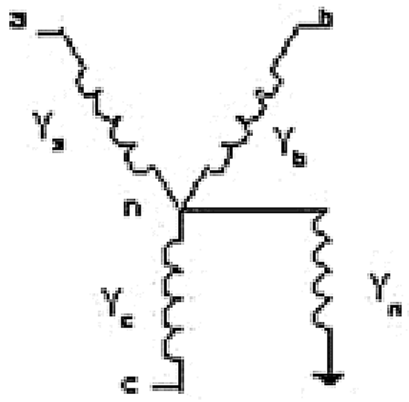

Figure 4. Shunt fault simulation

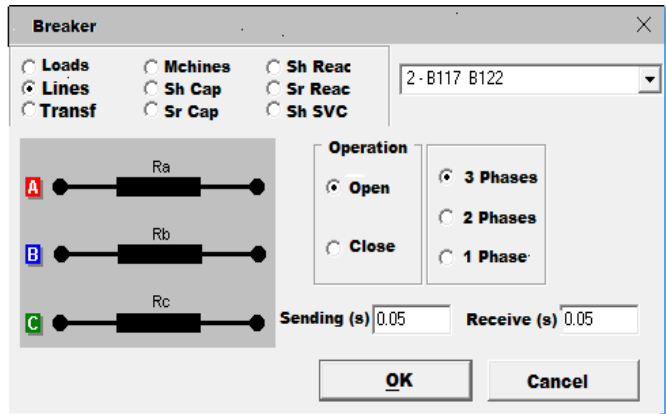

Figure 5. Breaker switching configuration

The impedance measured by the phase comparator are obtained from voltages obtained from the sending and receiving end of the line selected, and the complex currents registered on it. Each point corresponds to each time step $\mathrm{k}$ on the simulation. The expressions used for the line to line impedance measured are: 


$$
\underline{Z}_{i j}(k)=\frac{\underline{V}_{i j}(k)}{\underline{I}_{i}(k)-\underline{I}_{j}(k)}
$$

and for the phase to ground, impedance measured.

$$
\underline{Z}_{p h}(k)=\frac{\underline{V}_{i}(k)}{\underline{I}_{i}(k)+\underline{k}_{0} \underline{I}_{0}(k)}
$$

where,

$$
\underline{k}_{0}=\frac{\underline{Z}_{0 L}-\underline{Z}_{1 L}}{\underline{Z}_{1 L}}
$$

The voltages for each node $\mathrm{i}$ and stage $\mathrm{k}$ are obtained from the voltage sub-vector.

$$
\left[\underline{V}_{i}\right]^{a b c}=\left[T_{S}\right]\left[\underline{V}_{i}\right]^{012}
$$

From the network voltage vector, the $\mathrm{k}^{\text {th }}$ stage solution is:

$$
\left[\underline{V}_{B U S}\right]^{012}=\left[\underline{Z}_{B U S}\right]^{012}\left[\underline{I}_{B U S}\right]^{012}
$$

and the currents for a line SR by the matrix relation.

$$
\left[\begin{array}{l}
{\left[\underline{I}_{S}\right]^{a b c}} \\
{\left[\underline{I}_{R}\right]^{a b c}}
\end{array}\right]=\left[\underline{T}_{S}\right]\left[\begin{array}{l}
{\left[\underline{Y}_{S S}\right]^{012}\left[\underline{Y}_{S R}\right]^{012}} \\
{\left[\underline{Y}_{R S}\right]^{012}\left[\underline{Y}_{R R}\right]^{012}}
\end{array}\right]\left[\begin{array}{l}
{\left[\underline{V}_{S}\right]^{012}} \\
{\left[\underline{V}_{R}\right]^{012}}
\end{array}\right]
$$

The characteristics of the mho elements in the complex plane define the distance element selected, the reach and the polarization. Figure 6 shows the optional selections, this can be any combination, including only one or all of them.

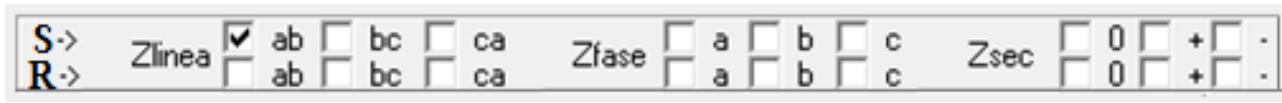

Figure 6. Optional distance elements

Table 2 shows the six possible polarizations implemented on this program version. They are: the self-polarized, two combined polarizations and three simple polarizations. The last one uses the positive sequence voltages, and the others considers a line or phase voltages. It shows only two of the six distance units for each case.

Table 2. Polarizations implemented

\begin{tabular}{ccccccc}
\hline Unit & $\underline{S}_{2}$ Self & $\underline{S}_{2}$ Combined & $\underline{S}_{2}$ Combined & $\underline{S}_{2}$ Simple & $\underline{S}_{2}$ Simple & $\underline{S}_{2}$ Sec pos \\
\hline $\mathrm{AB}$ & $\underline{V}_{a b}$ & $\underline{V}_{a b}+k_{p} \underline{V}_{c} \angle-90^{0}$ & $\underline{V}_{a b}+k_{p} \underline{V}_{c a} \angle-120^{0}$ & $k_{p} \underline{V}_{b c} \angle-120^{0}$ & $k_{p} \underline{V}_{c} \angle-90^{0}$ & $\underline{V}_{a b 1}$ \\
$\mathrm{~A}$ & $\underline{V}_{a}$ & $\underline{V}_{a}+k_{p} \underline{V}_{b c} \angle+90^{0}$ & $\underline{V}_{a}+k_{p} \underline{V}_{c} \angle-120^{0}$ & $k_{p} \underline{V}_{b} \angle-120^{0}$ & $k_{p} \underline{V}_{b c} \angle+90^{0}$ & $\underline{V}_{a 1}$ \\
\hline
\end{tabular}

The software simulates a memory polarization with positive sequence voltage [25]. As Shown in (35) determines the polarization voltage for each time step.

$$
V_{1 M E M[k]}=\alpha V_{1[k]}+(1-\alpha) V_{1 M E M[k-1]}
$$


where,

$V_{1 M E M[k]}$ is the present memorized positive sequence voltage, which must be used in the step $\mathrm{k}$ as polarization voltage.

$V_{1[k]}$ is the actual positive sequence voltage value.

$V_{1 M E M[k-1]}$ is the previous memorized positive sequence voltage value.

$\alpha$ is the memory time constant.

Then for each line selected, with a mho phase comparator, the $\underline{S}_{l}, \underline{S}_{2}, \underline{A}_{p}$ and $\underline{B}$ values are calculated for each distance unit protection selected, for the reach given to each zone, the polarization of the unit and voltage and current output values given to the program use the relations (32) to (34). The complex impedance curves measured, and the characteristics zones in a mho phase comparator are in a complex plane. Figure 7 shows the impedance measured modules curves, resistance or reactance versus time and $\theta$ angles versus time using an alternative form. All these curves can be shown from the beginning of the simulation up to an elected time, while the characteristics of the mho units, drawing the variable values of the selected instant time. Every point of the curves and graphics in the complex plane relates the corresponding instant time of the simulation. The complex model accuracy characteristics of the mho units may be tested with regarding the $\theta$ vs time curves, based straightforward in the operating principle of phase comparison considering the input signals of these units. All points of the mho characteristics and impedance measured curves are subtracted from the line impedance $\underline{Z}_{l}$ for protections are on the receiving end of a line. Thus the graphics for these protections take as origin of the complex planethe value of the impedance of the line and are rotated 180 degrees.

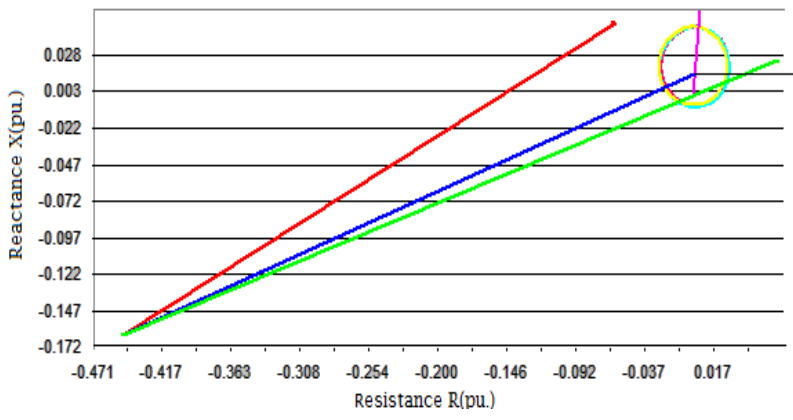

(a)

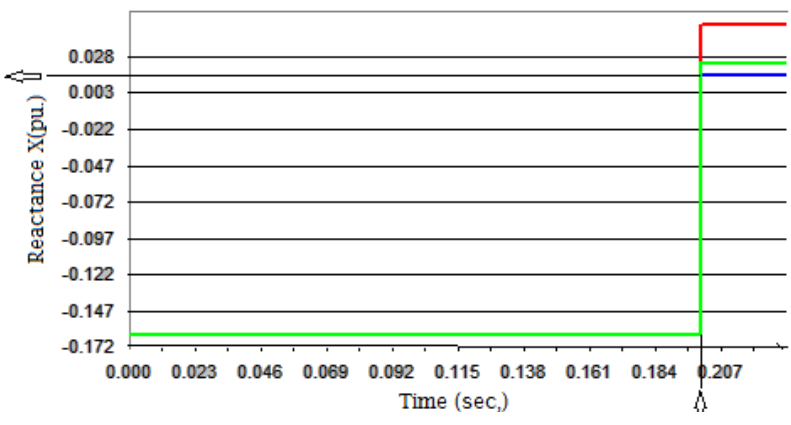

(b)

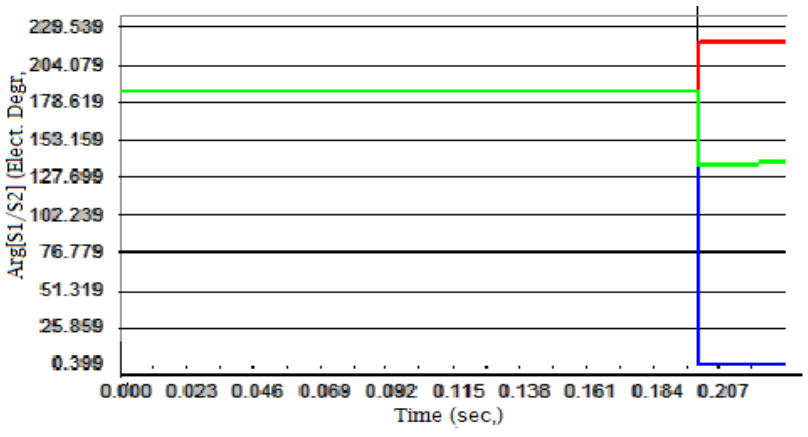

(c)

Figure 7. Complex plane and related reactance curve, (a) impedance measured curves and mho characteristcs, (b) reactance versus time, (c) arg. $\left(\underline{S}_{1} / \underline{S}_{2}\right)$ versus time 


\section{STUDY CASES}

Test system IEEE 24 nodes was used for the following modeling examples in a mho type phase comparator [29].

Case 1: The total initial load of the system is $2850 \mathrm{MW}$ and $580 \mathrm{MVAR}$. Load increases with the voltage stability in proportion to their initial values obtaining a total load, which is 1,603 times greater than the initial, for a critical network condition. The smallest eigenvalue was 0.0033 determined by a modal analysis. The node B106, the line L35 and the generator G10 were the elements with highest participation factor, for this operation mode. Figure 8(a) shows the voltage vs active power curve in the critical node B106. Lines protections located in the L20 with higher percentage of active power transfer exhibit the impedance measured curve by them, from the initial condition to the critical point, in Figure 8(b). The protections at the receiving end are shown, because its third zone is the closest to the critical impedance point. The mho characteristics in this case are three forward zones, first 80, second 120 and third $250 \%$ of the line L20 and a third inverted zone with $120 \%$ of impedance on the line L20, all with self-polarization. The impedance measured at the critical point is reduced to $10.6 \%$ from its initial value. This point is near to the operation zones in the graphic and consequently the probability operation of this line is higher, caused by a fluctuation.

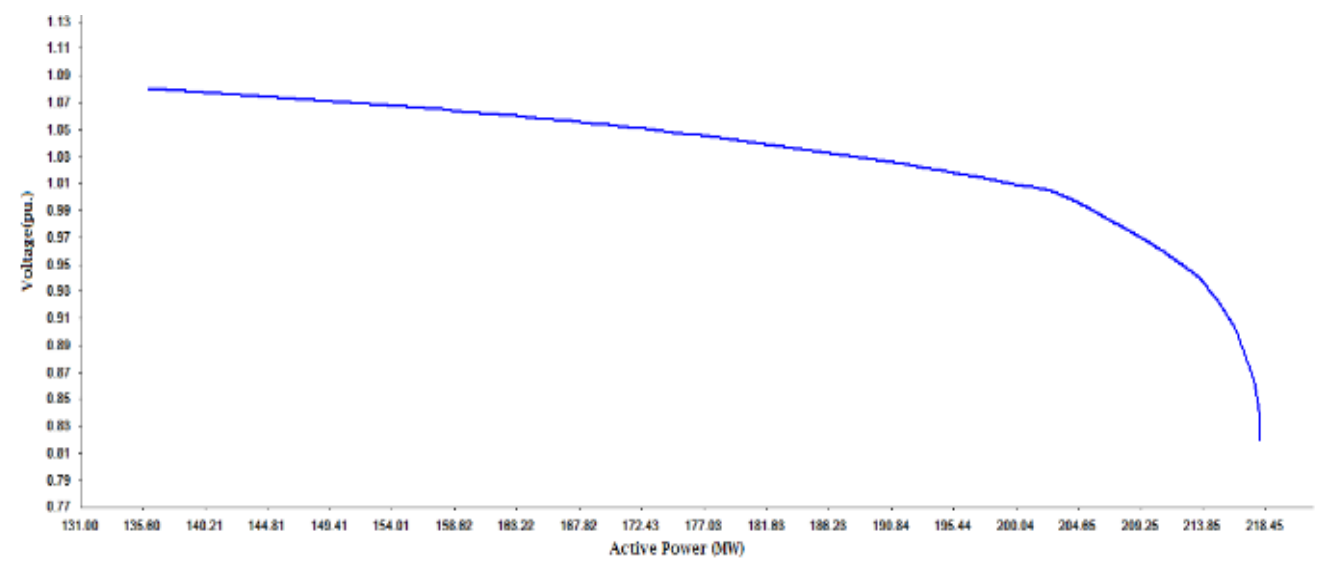

(a)

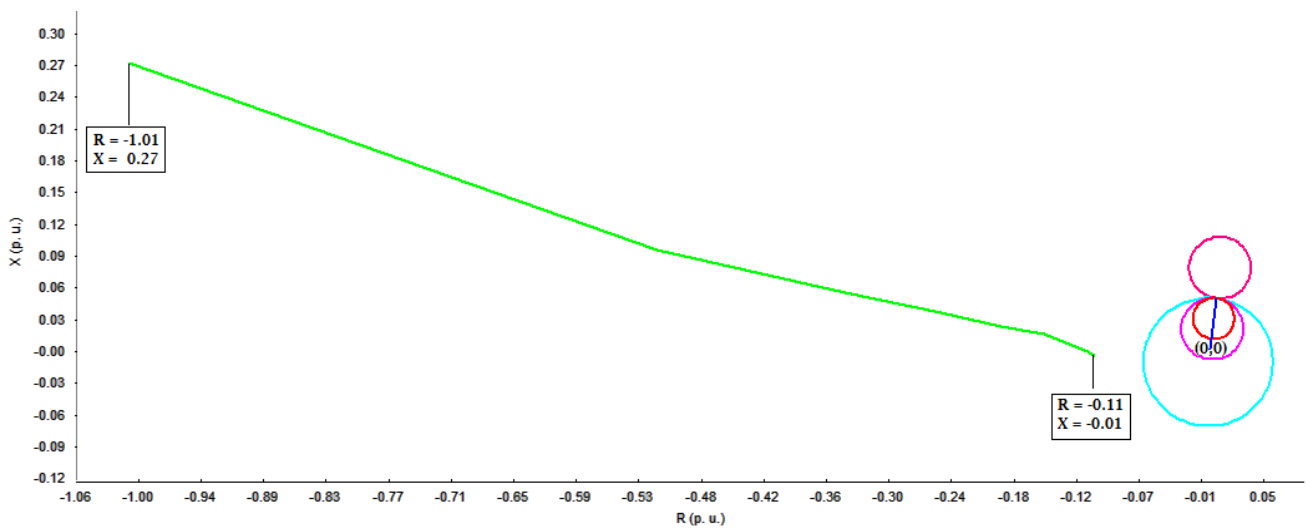

(b)

Figure 8. Static voltage stability curves, (a) critical node V vs P curve, (b) complex plane. Impedance measured curve and mho characteristics

Case 2: A fault line to line ground $(\mathrm{BC})$ at the receiving end of the line L20 is analyzed with the first and second mho operation zones but with a positive memory sequence polarization in all time intervals [29], point $\mathrm{F}$. The memory effect is shown on the displacement of mho operation zones around the complex origin plane. The successive sequence changes are onset to fail at $0.2 \mathrm{sec}$, faulted phases opening at 0.27 seconds and a successful re-closure at 0.37 seconds. Figure 9(a) describe all phase and line protections overlap in both zones under pre-fault conditions. Also all load impedance measured are equal as is shown in the straight line from the receiving end line to load impedance value. At 0.2 seconds fault onset, three-line protections 
practically overlap and therefore two zones are only witnessed while the six phase operation zones are offset one from another Figure 9(b). All zones operate except the two zones of phase A, because all impedance measured are very close from the end of the line. Twelve zones are discriminated very subtly at 0.27 seconds end of the fault time interval and all zones have been moved with respect the beginning of the fault as is shown in Figure 9(c). All zones remain triggered except the two zones of phase A. The voltage and current values in all phases and memory output voltages have variations in the time interval.

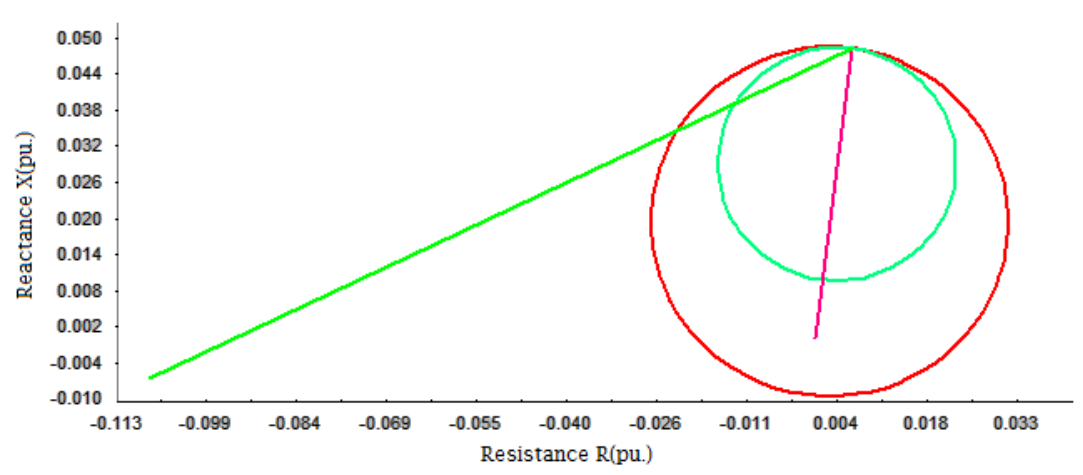

(a)

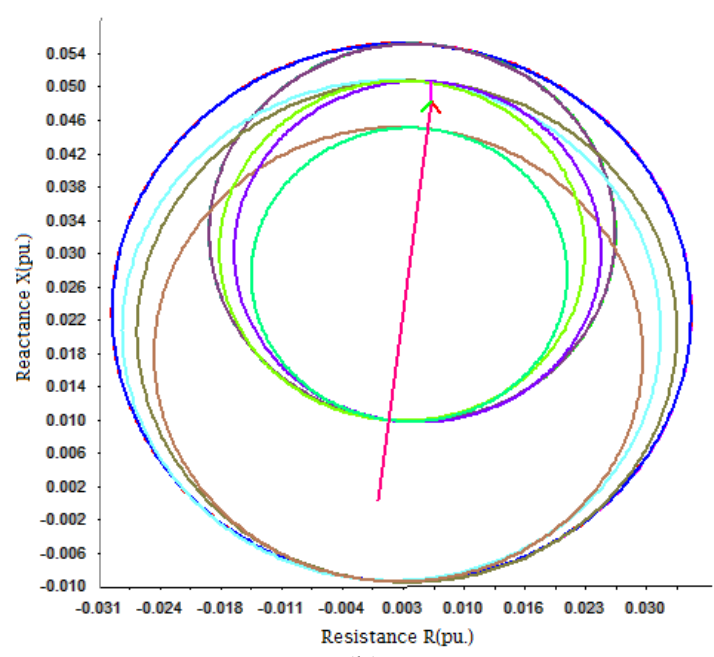

(b)

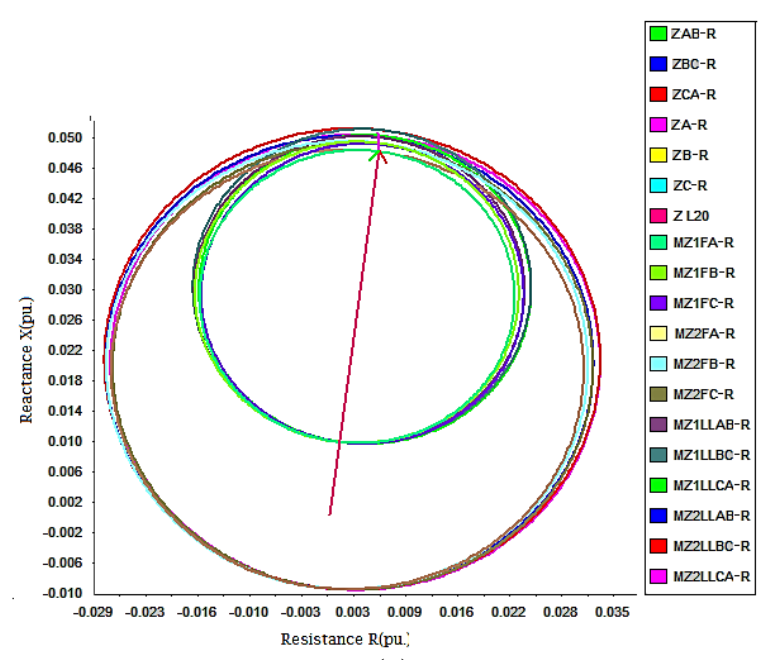

(c)

Figure 9. Impedance measured from receiving end before fault and mho operation zones in fault conditions, (a) 0 to $0.20 \mathrm{~s}$, (b) fault $0.20 \mathrm{~s}$, (c) faults $0.27 \mathrm{~s}$

$\mathrm{AB}, \mathrm{CA}$ and $\mathrm{A}$ zones are shown in Figure 10(a) when $\mathrm{B}$ and $\mathrm{C}$ phases open at 0.27 second and impedance measured of these three zones are outside of their areas. The same six areas have reduced their dimensions at 0.37 second Figure 10(b) and their impedance measured are out of their reach. Phases B and C are closed at 0.37 second and balanced conditions are restored in Figure 10(c). The two zones with the six protections coincide but they are displaced from the end of the line, because voltages are not constants. Also, these voltages and frequency variations produce output memory voltages variations. The adaptive short or not memory vs long memory have been used by manufacturer to avoid mho miss-operation in these conditions $[24,33]$.

The $\theta$ curves of twelve operation zones with polarized positive sequence using memory in the last example are shown in Figure 11 for the interval from 0.15 to 0.4 seconds. The mho zones behavior in the complex plane from Figures 11 and 12 agree with results given by $\theta$ curves in the pre-fault, fault and post-fault conditions. The mho zones displacement equations in the complex plane are tested again in this example conditions.

For the last example, all second zone positions of phase A are shown in Figure 12, each 0.01 second from 0 to 1 second. The characteristic at 0.00 second, delimits top and right ends of the variation region in the complex plane. The characteristic at $0.20 \mathrm{sec}$ at beginning of fault delimits the left and bottom ends of the region. 


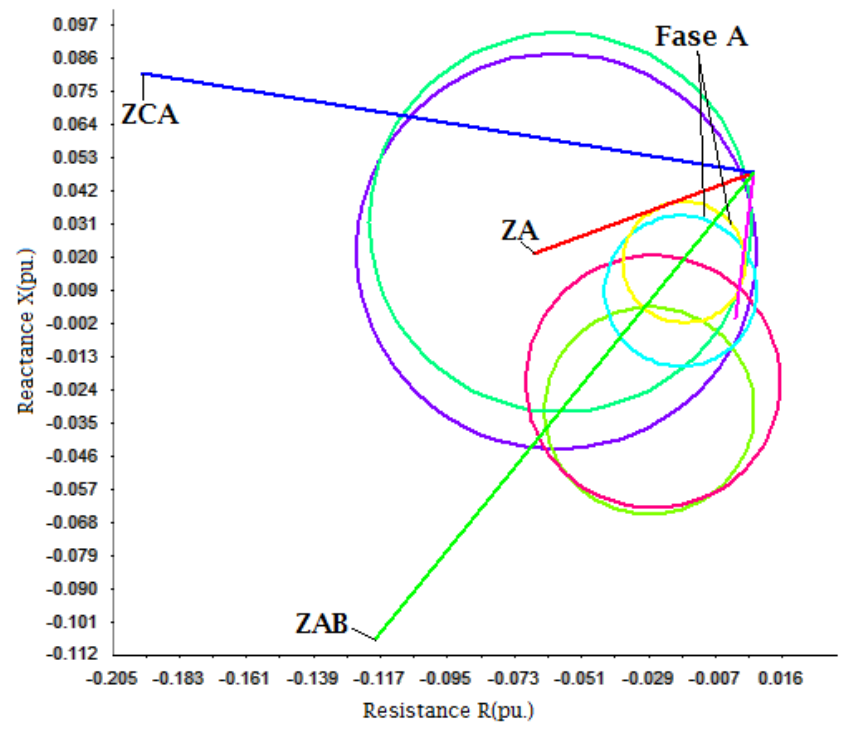

(a)

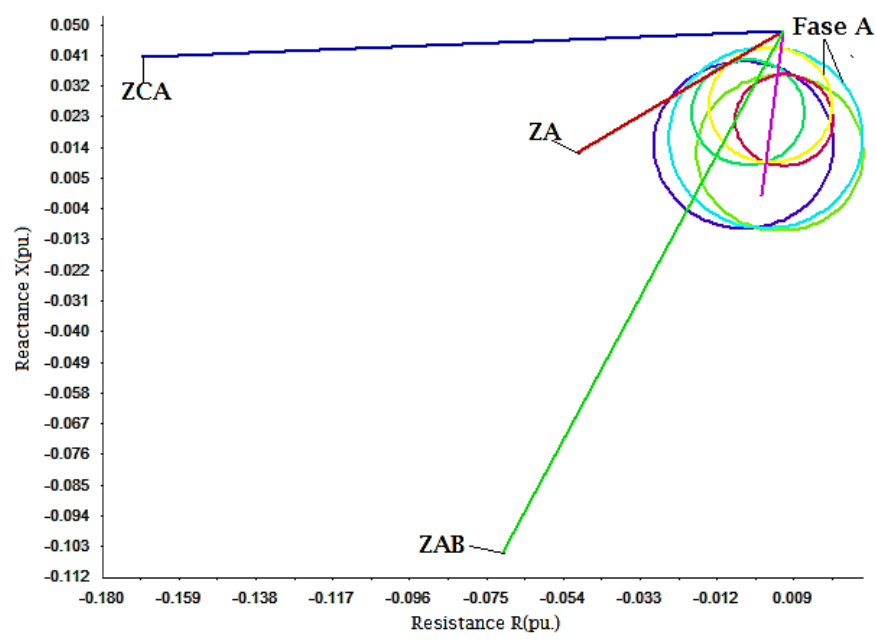

(b)
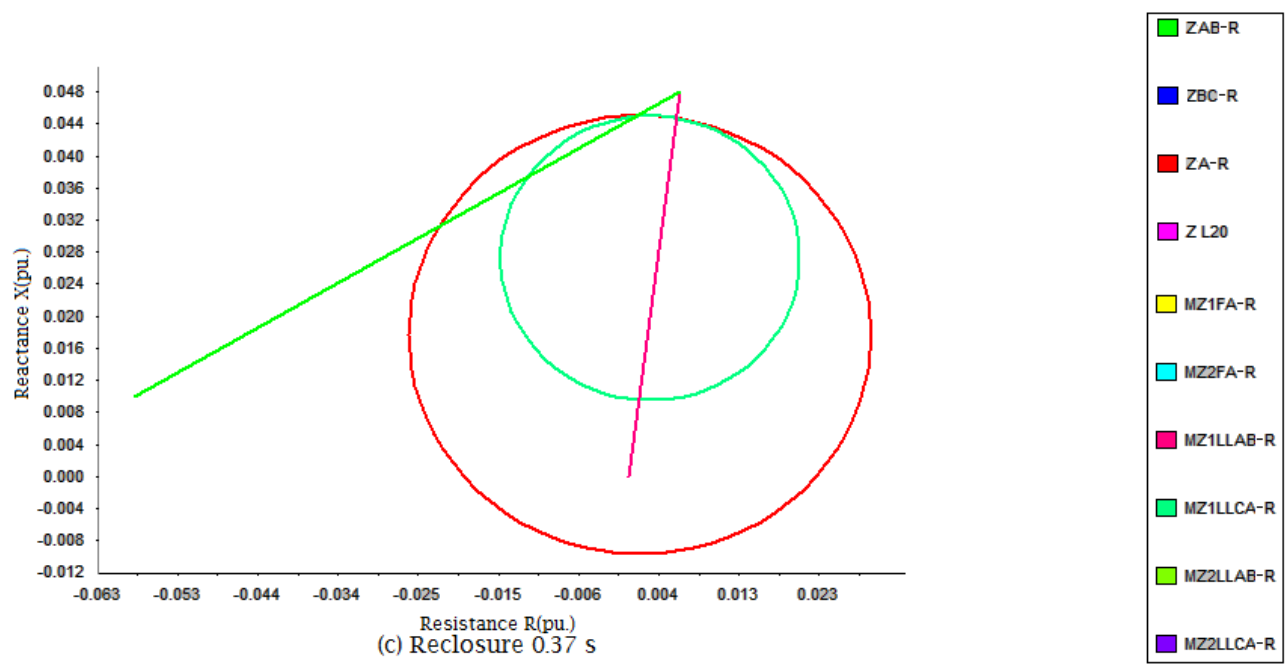

(c)

Figure 10. Mho operation zones with B and C open and post fault conditions, (a) BC open $0.27 \mathrm{~s}$, (b) $\mathrm{BC}$ open $0.37 \mathrm{~s}$, (c) reclosure $0.37 \mathrm{~s}$ 


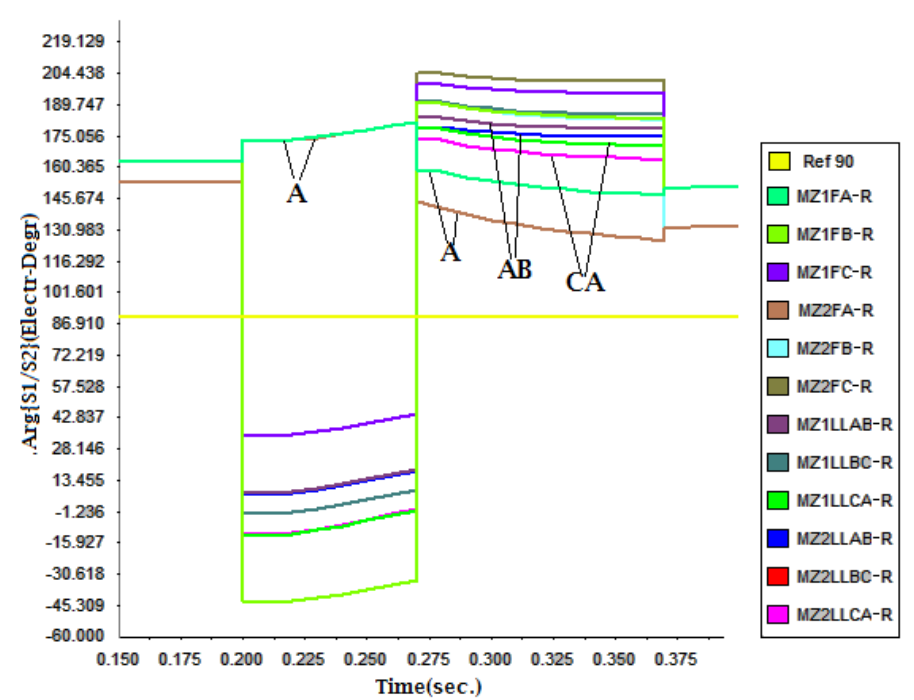

Figure 11. $\theta$ curves in a mho operation zones

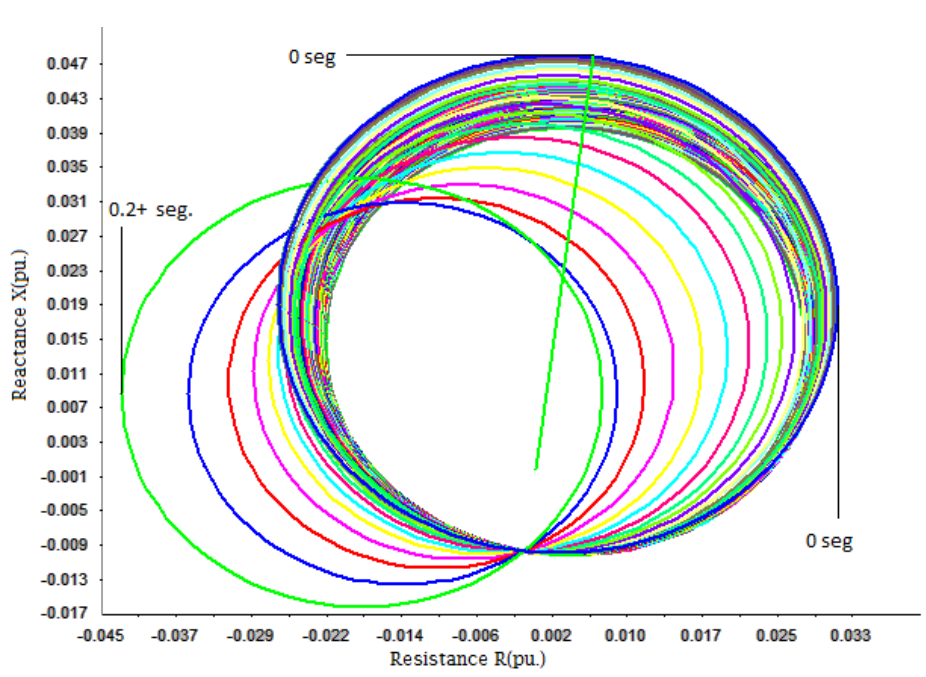

Figure 12. Phase a displacement in the complex plane, from 0 to $1 \mathrm{~s}$

\section{CONCLUSION}

This paper modeled a mho phase comparator in the complex plane using the results of a static voltage stability program. This simulation detects unit distance elements with impedance values measured near their reach threshold. The critical system elements detected can be used for contingency analysis in dynamic disturbances. The mho phase comparators dynamic behavior can be modeled using the method described for the most important disturbances types, with the results of a mid-term stability digital program as input. The polarization effects simulation, type and memory usage, were tested successfully in two numerical study cases. Short circuit analysis achieves similar results for a fault during a period as a dynamic simulation case. The model used in the complex plane was tested considering the argument means (S1/S2) curves, designated as $\theta$ angle curves. These curves can be exploited other applications analysis in a phase comparator and the corresponding models in the complex plane. This comparative analysis is readily applicable to the amplitude comparator curves through the input signals modular ratio.

\section{REFERENCES}

[1] C. Da Costa, et al., "A Methodology for Distance Relay Modeling," IEEE Latin America Transactions, vol. 16, no. 5, pp. 1388-1394, 2018. 
[2] M. R. Barzegar, and A. Foroud, "Performance evaluation of distance relay in the presence of hybrid SFCL," IET Science Measurement and Technology, vol. 12, no. 5, pp. 581-593, 2018.

[3] W. D. Humpage and S. P. Sabberwal, "Developments in-phase-comparison techniques for distance protection," Proceedings of the Institution of Electrical Engineers, vol. 112, no. 7, 1965, pp. 1383-1394.

[4] G. W. Stagg and A. H. El-Abiad, "Computer Methods in Power System Analysis," New York: McGraw-Hill Book Company, 1968.

[5] L. Jackson, et al., "Distance protection: optimum dynamic design of static relay comparators," Proceedings of the Institution of Electrical Engineers, vol. 115, no. 2, 1968, pp. 280-287.

[6] A. Manori, et al., "Advance compensated mho relay algorithm for a transmission system with shunt flexible AC transmission system device," Electric Power Components and Systems, vol. 42, no. 16, pp. 1802-1810, 2014.

[7] G. T. Vuong and G. Paris, "Rule-based relay modeling for transient-stability studies," IEEE Transactions on Power Systems, vol. 3, no. 3, pp. 1306-1309, 1988.

[8] L. P. Cavero, "Computer-aided evaluation and application of distance relays," 1993 Fifth International Conference on Developments in Power System Protection, York, UK, 1993, pp. 199-202.

[9] S. J. Zubić, et al., "Probabilistic assessment of new time-domain distance relay algorithms," Electric Power Systems Research, vol. 119, pp. 218-227, 2015.

[10] Do, Dinh-Thuan, and Minh-Sang V. Nguyen, "Enabling relay selection in non-orthogonal multiple access networks: direct and relaying mode," TELKOMNIKA (Telecommunication, Computing, Electronics and Control), vol. 18, no. 2, pp. 587-594, 2020.

[11] V. Cook, "Generalised method of assessing polarising signals for the polarised mho relay," Proceedings of the Institution of Electrical Engineers, vol. 122, no. 5, 1975, pp. 497-500.

[12] A. B. Shah, et al., "Mho Relay for Protection of Series Compensated Line," 2009 IEEE Toronto International Conference Science and Technology for Humanity (TIC-STH), Toronto, ON, 2009, pp. 648-651.

[13] Abd Almuhsen, Tahseen Ali, and Ahmed Jasim Sultan, "Coordination of directional overcurrent and distance relays based on nonlinear multivariable optimization," Indonesian Journal of Electrical Engineering and Computer Science (IJEECS), vol. 17, no. 3, pp. 1194-1205, 2020.

[14] S. J. Zubić, and M. B. Djurić, "A distance relay algorithm based on the phase comparison principle," Electric Power Systems Research, vol. 92, pp. 20-28, 2012.

[15] H. J. Altuve, et al., "Simulación digital de relevadores analógicos de distancia a nivel de esquema de comparación," Parte I X Reunión de Verano de Potencia del IEEE Sección México (RVP-97), Acapulco, Gro., México, 1997.

[16] H. J. Altuve, et al., "Simulación digital de relevadores analógicos de distancia a nivel de esquema de comparación," Parte II: Programa Universidad Autónoma de Nuevo León, Facultad de Ingeniería Mecánica y Eléctrica, México, 1997.

[17] R. C. Dos Santos, and E. C. Senger, "Transmission lines distance protection using artificial neural networks," International Journal of Electrical Power and Energy Systems, vol. 33, no. 3, pp. 721-730, 2011.

[18] M. D. Zadeh, et al., "FPAA-based mho distance relay considering CVT transient supervision," IET generation, transmission and distribution, vol. 3, no. 7, pp. 616-627, 2009.

[19] S. Raman, et al., "An adaptive fuzzy mho relay for phase backup protection with in feed from STATCOM," IEEE Transactions on Power Delivery, vol. 28, no. 1, pp. 120-128, 2013.

[20] A. Ghorbani, et al., "Operation of synchronous generator LOE protection in the presence of shunt-FACTS," Electric Power Systems Research, vol. 119, pp. 178-186, 2015.

[21] A. Manori, et al., "SVM based zonal setting of Mho relay for shunt compensated transmission line, ” International Journal of Electrical Power and Energy Systems, vol. 78, pp. 422-428, 2016.

[22] L. T. Guajardo, and A. C. Enríquez, "Enhanced performance for distance relays due to series capacitors in transmission lines," Electric Power Systems Research, vol. 109, pp. 20-31, 2014.

[23] Guajardo, et al., "Error compensation in distance relays caused by wind power plants in the power grid," Electric Power Systems Research, vol. 106, pp. 109-119, 2014.

[24] H. J. Altuve, and E. O. Schweitzer, "Modern solutions for protection, control, and monitoring of electric power systems," Schweitzer Engineering Laboratories, 2010.

[25] S. Zamora, "Flujo de carga N-R acoplado rápido con técnicas para orientar el análisis en caso de divergencia," Tesis de Master en Ciencias, UCLV, Santa Clara, 1999.

[26] J. Arrillaga, and N. R. Whatson, "Computer modelling of Electrical Power Systems," New York: John Wiley and Sons Ltd., Second Edition, 2001.

[27] P. Kundur, "Power System Stability and Control," New York: Mc Graw Hill, Inc., 1993.

[28] V. Ajjarapu, and C. Christy, "The continuation power flow: A tool for steady state voltage stability analysis, " IEEE Transactions on Power Systems, vol. 7, no. 1, pp. 416-423, 1992.

[29] Z. García Sánchez, "Metodología para estudios estáticos de la estabilidad de tension," Tesis de Doctorado en Ciencias Técnicas, UCLV, Santa Clara, Cuba, 2011.

[30] Z. Garcia, et al., "Voltage collapse point evaluation considering the load dependence in a power system stability problem," International Journal of Electrical and Computer Engineering (IJECE), vol. 10, no. 1, pp. 61-71, 2020.

[31] C. Cañizares, "Voltage stability assessment, procedures and guides," IEEE/PES Power System Stability Subcommittee, Special Publication, 2001.

[32] E. G. Gate, et al., "Time frame notion and time response of the methods in transient, mid-term and long-term stability programs," IEEE Transactions on Power Apparatus and Systems, vol. 103, no. 1, pp. 143-151, 1984.

[33] L. Wang, and E. Price, "New High-speed Microprocessor Distance Relaying for Transmission Lines," POWERCON '98. 1998 International Conference on Power System Technology. Proceedings (Cat. No.98EX151), Beijing, China, vol. 2, 1998, pp. 1143-1147. 


\section{BIOGRAPHIES OF AUTHORS}
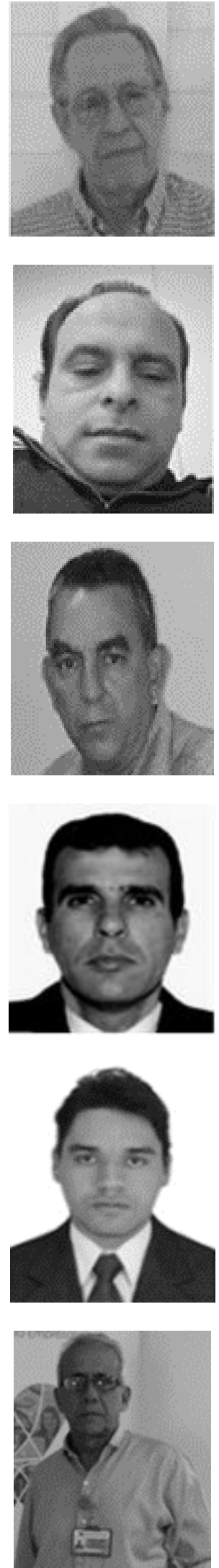

José Antonio González-Cueto Cruz. Electrical Engineer, Master of Science, Doctor of Technical Sciences, Senior Associate Professor, Center for Electroenergetic Studies (CEE), Central University "Marta Abreus" de las Villas. E-mail: jcueto@uclv.edu.cu.

Zaid Garcia Sanchez was born in Santa Clara, Cuba on November 11, 1978. Receive the B.S inElectrical Engineer from the Universidad Central de las Villas, Cuba in 2002. Master of Science in Electric Power Systems in 2004 and Dr. C (Ph.D) degree fron the Universidad Central de las Villas, Cuba in 2011. Full Professor, Center for Energy and Environment Studies (CEEMA), University of Cienfuegos E-mail: zgarcia@ucf.edu.cu. His area of interest includes power systems modelling and stability, Renewable Energy Sources and Smart Grid. https://orcid.org/0000-0003-1989-9362

Gustavo Crespo Sánchez Electrical Engineer, Master of Science, Assistant Professor, Center for Energy Studies and Environment (CEEMA), University of Cienfuegos. E-mail: gcsánchez@ucf.edu.cu.

Hernan Hernández Herrera Cienfuegos, Cuba on July 7, 1976. Received the B.S degree in Mechanical Engineering from the Universidad de Cienfuegos, Cuba in 2000. M. Sc. in applied mechanics from the Universidad de Cienfuegos, Cuba 2005. Received the Dr.C. (Ph.D.) degree from the Universidad de Cienfuegos, Cuba, in 2006. Currently is professor of plant in the faculty of mechanical engineering in the University Simón Bolívar, Colombia. His area of interest includes Applied Mechanics, Energy Efficiency and Renewable Energy Sources.

Jorge Iván Silva Ortega is Electrical and Electronic Engineer, Master in Electrical Engineering (2013) from the Universidad del Norte, Colombia. PhD student in Engineering at the Universidad Pontificia Bolivariana in Medellín, Colombia. Professor and researcher of the Universidad de la Costa, Colombia. joined to Research Group on Energy Optimization (GIOPEN). His research interests include: electrical power systems, electrical engineering education; planning, operation and control of power systems; and intelligent electrical networks. https:/orcid.org/0000-0002-7813-0142.

Leonel Martínez-Díaz, Cienfuegos, Cuba on July 19, 1954. Received the B.S degree in Mechanical Engineering from the Universidad de Cienfuegos, Cuba in 1980. Received the Dr.C. (Ph.D.) degree from the Universidad de Cienfuegos, Cuba, in 2000. Currently is professor of plant in the faculty of mechanical engineering in the University Simón Bolívar, Colombia.

His area of interest includes Thermo fluid, Energy Efficiency and Renewable Energy Source. 